\title{
UNA NUEVA PINTURA DE PETER THIJS IDENTIFICADA EN LA COLEGIATA DE SANTA GERTRUDIS DE NIVELLES (VALONIA)
}

Tenida en la actualidad como anónima, y atribuida a Gaspar de Crayer en documentos de principios del siglo XX, la pintura de la Colegiata de Nivelles es comparable en composición, modelos, factura y sentido del color con otras obras de Peter Thijs. El pintor recurre, como fue su costumbre, a Van Dyck, a la vez que se distancia de las representaciones más frecuentes de este episodio de la vida de Santo Domingo, cuyo fervor creció en Amberes por impulso de dominicos y jesuitas en la primera mitad del siglo XVII.

Palabras clave: Thijs; Nivelles; Santo Domingo; Van Dyck; Rosario; Pintura.

Considered today as anonymous and attributed in a 1925 document to Gaspar de Crayer, the painting at Nivelles reveals the stylistic features of Peter Thijs: composition, models, handling and color are comparable to other works by his hand. As usual, Thijs has recourse to the paintings of Van Dyck, while eluding the more habitual depictions of this episode from the life of Saint Dominic, fervor for whom grew in Antwerp during the first half of the $17^{\text {th }}$ century, due promotion by Dominicans and Jesuits.

Key words: Thijs; Nivelles; Saint Dominic; Van Dyck; Rosary; Painting.

Ya se viene subrayando últimamente la valía de este pintor al que se ha prestado poca atención en el pasado". Considerado hasta fechas recientes como un "modesto emulador de Van Dyck", las obras de Peter Thijs (Amberes, 1624-1677) han sido atribuidas durante años a otros pintores de mayor fama, como Van Dyck, Thomas Willeboirts Bosschaert, Jan Boeckhorst o Erasmus Quellinus. Afortunadamente en los últimos tiempos se han ido identificando y restituyendo obras suyas que van dando prueba de la extrema calidad de su producción, tanto en escenas religiosas y mitológicas, como en retratos. Es el caso de un Lavatorio de la parroquia de San Pedro y San Pablo de la ciudad croata de Bribir, que se tenía por obra de Palma el Joven ${ }^{3}$; de un Santo martirizado en presencia de San Antonio de Padua de colección privada de Bruselas atribuido a Van Dyck $^{4}$, de una Cacería del jabalí de Calidonia en colaboración con Jan Fyt del Museo Ringling de Sarassota que contaba con un largo historial de atribuciones (de Rubens a Jan Boeckhorst y Quellinus) y cuya correspondencia con una pintura inventariada en la colección de Diego Duarte en Amberes (1682), con especificación de su autoría en las figuras, permitió su restitución a Thijs ${ }^{5}$. También le han sido asignados un Cazador con perros del Museo Cumming de Florida en colaboración con Peter Boel, anteriormente atribuido a Erasmus Quellinus y Jan $\mathrm{Fyt}^{6}$, y $E l$ Tiempo y las Parcas del Museo de Ginebra, que se tenía por obra de Théodoor van Thulden? Entre los retratos vale la pena subrayar la identificación de su Autorretrato en el Museo de Bru-

1 J. Douglas Stewart, "Peter Thijs (1624-1677). Rediscovering a scarcely known Antwerp painter", Apollo, vol. CXLV, 1997, pp. 37-43.

2 M. L. HaIrs, Dans le sillage de Rubens. Les Peintres d'Histoire anversois au XVIIème siècle, Lieja, 1977, p. 78; Gerson Ter KuILE, Art and Architecture in Belgium 1600-1800, 1960, p. 142. 120 .

3 R. TомIC, "Prijedlog za Pietera Thysa: Pranje nogu in Bribiru ", Perystil, XXXVIII, Zagreb, 1995, pp. 117-

${ }^{4}$ Lienzo, $245 \times 170 \mathrm{~cm}$. Véase A. BALIS, "Van Dyck: Some Problems of Attributions", en Van Dyck 350, Studies in the History of Art 46, Center for Advanced Studies in visual Arts, Symposium Papers, XXVI, Washington, 1994, p. 180, n. 13.

5 A. BALIs, "Pieter Thijs, Pepijn en een derde pseudo-Boeckhorst", en Jan Beckhosrt Medewerken van Rubens, Cat. Exp. Amberes, Rubenshuis, 1990, pp. 98-108.

${ }^{6}$ Lienzo, 188,3 $\times 257,9 \mathrm{~cm}$. Cumming Museum and Gardens, Florida, Inv. n. ${ }^{\circ} 1106$ [véase H. Vlieghe, "Een wazige kijk op Erasmus II Quellin. Bedenkingen bij recent onderzoek", Jaarboek van het Koninklijk Museum voor Schone Kunsten, Antwerpen, 1993, p. 305, fig. 16].

7 VAn Gelder, in Oud Holland 64, 1949, p. 45 (como Th. Van Thulden); D. Maufort, "Une Vanité avec trois Parques au Musée d'Art et d'Histoire de Genève et l'œuvre du peintre anversois Pieter Thijs (1624-1677)", Genava, Revue d'Histoire de l'Art et d'archéologie du Musée d'Art et d'Histoire de Genève, XLIX, 2001, pp. 3-15 (como P. Thijs). 
selas $^{8}$, y el de un Monje agustino, de colección privada española, que se tenía por obra de Van Dyck y que fue dado a conocer recientemente en España ${ }^{9}$.

El trabajo más completo sobre el maestro se debe a Danielle Maufort, quien trató su producción religiosa y mitológica en tesis de licenciatura de la Universidad de Lovaina, defendida en 1986 y lamentablemente inédita ${ }^{10}$. Previamente contábamos con el capítulo que le dedicó M. L. Hairs en su titánico trabajo sobre los pintores satélites de Rubens ${ }^{11}$. Los artículos citados a lo largo de este estudio han contribuido a esclarecer el estilo y la personalidad del pintor. No obstante, algunas obras no gozan hoy día del consenso de la crítica. Es el caso de un San Francisco en éxtasis de colección española, adquirido como obra de Gaspar de Crayer y atribuido a Thijs en ventas anteriores. J. Douglas Stewart propuso a Thomas Willeboirts Bosschaert como su autor, atribución que apoyó M. Díaz Padrón en artículo reciente, pero que no recoge el estudio monográfico de Axel Heinrich publicado en $2003^{12}$. Otro ejemplo es El Tiempo y la diosa de la Fortuna del Palacio de Sanssouci y la versión del Museo de Grenoble, que constan en la monografía citada de Willeboirts, a pesar de las opiniones en pro de Peter Thijs, criterio este último que compartimos ${ }^{13}$.

En cualquier caso, los documentos y las obras demuestran la consideración de la que gozó Peter Thijs en su época, al testimoniar que recibió encargos de nobles y príncipes, como el Archiduque Leopoldo Guillermo ${ }^{14}$, a quien retrató hacia $1649^{15}$, y para quien realizó, según

${ }^{8}$ D. MAufort, "Een zelfportret van Peeter Thijs de Oude (1624-1677) in de Koninklijke Musea voor Schone Kunsten van België”, Bulletin des Musées Royaux des Beaux Arts de Belgique, 1994/1-4 - 1995/1-5, Bruselas, 2000, pp. 103-111.

9 M. DíAz PAdRón, "Un retrato de monje agustino con atribución a Van Dyck, restituido a Peter Thijs”, Goya, n. ${ }^{\text {os }} 307-308,2005$, pp. 261-264.

${ }^{10}$ D. Maufort, De Antwerpsche kunstschilder Peeter Thiijs de Oude. Een enadering aan de hand van zjn historiestuken, Tesis de Licenciatura inédita de la Universidad Católica de Lovaina, 1986.

11 M. L. HaIrs, Dans le sillage de Rubens. Les Peintres d'Histoire anversois au XVIlème siècle, Lieja, 1977, pp. 265-276.

12 San Francisco y el ángel músico, lienzo, $113 \times 80,7$ cm. Colección privada española. Galería D. H. Katz, 1942, 1961 (como Peter Thijs); Christie's, Londres, 17-IV-2002, n. ${ }^{\circ} 19$ (como Gaspar de Crayer); Wolfgang Heinrich SAVELSBERG, Die Dartellung des Hl. Franziskus van Asissi in der flämischen Malerei und Graphiek des späten 16 und 17. Jahrhundert, Roma, 1992, n. 117 (como anónimo); J. Douglas STEwart, "Thomas Willeboirts Bosschaert and Pieter Thijs: A Tale of Two Tangled Antwerp Painters; with an Excursus on Van Dycks's St. Felix of Cantalice", in Van Dyck 1599-1999, Conjectures \& Refutations, Brepols, 2001, p. 271, fig. 1 (como Thomas Willeboirts Bosschaert); A. HeINRICH, Thomas Willeboirts Bosschaert (1613/14-1654). Ein Flämische nachfolger Van Dycks, Turnhout, 2003, 2 vols. (no figura); M. DíAz PADRÓN, "Una nueva pintura de Thomas Willeboirts Bosschaert en España: San Francisco y el ángel músico", Boletín del Seminario de Estudios de Arte y Arqueología de la Universidad de Valladolid (en prensa) (como Thomas Willeboirts Bosschaert).

${ }^{13}$ El lienzo de Potsdam $(193 \times 207 \mathrm{~cm})$ fue destruido en la Segunda Guerra Mundial. El de Grenoble, $274 \times 195$ cm, Inv. MG 106 [véase F. BAUdoIn, "Antekeningen over Venus en Adonis tafeleren van Thomas Willeboirts Bosschaert en zijn invloed op de Hollandse schilderkunst", Oud Holland, XCVIII, 1984, p. 144 (como Willeboirts); MAUFORT, op. cit., 1986, pp. 181, 189-191, 192 (como Peter Thijs); H. VliegHe, Flemish Art and Architecture 1585-1700, New Haven, 1998, pp. 197-198, fig. 127 (como Peter Thijs); M. Destot, La Collection du musée de Grenoble: peintures des écoles du Nord, París, 1994, pp. 178-179 (como Willeboirts); MAUfort, op. cit., 2001, pp. 6-7 (como P. Thijs); Douglas Stewart, op. cit., 2001, p. 272 (como Thijs); HeInRICH, op. cit. 2003, cat. A30 y A30a (atribuye el de Potsdam a Willeboirts y el de Grenoble a su taller).

14 E. Duverger, Antwerpse kunstinventarissen uit de zeventiende eeuw, Bronnen voor de Kunstgeschiedenis van de Nederlanden, vol. VII, pp. 197-198, docs. 2051 y 2052.

${ }^{15}$ El Retrato del Archiduque Leopoldo Guillermo se conserva en el Museo de Viena (lienzo, $127 \times 86 \mathrm{~cm}$, Inv. n. 370 [M. L. HAIRS, op. cit. 1977, p. 265; A. BALIS e.a., De Vlaamse schilderkunst in het Kunsthistorisches Museum te Wenen, Amberes-Zürich, 1987, p. 287; M. Haja (ed.) e.a., Die Gemäldegalerie des Kunsthistorisches Museums in Wien, Verzeichnis der Gemälde, Wenen 1991, p. 120, il. T 436]. 
diseños de Jan van den Hoecke, parte de los cartones para los tapices de El Día y La Noche, y las Alegorías de los Meses, en colaboración con Thomas Willeboirts Bosschaert, Jan Brueghel el Joven y Adriaen Van Utrecht ${ }^{16}$. También prestó sus servicios a la casa de los Orange hacia 1650, retratando al príncipe Guillermo II en su Nombramiento como Stadhouder ${ }^{17}$. Su responsabilidad como Decano de la guilda de pintores de San Lucas de Amberes durante el año 1661-1662 habla también de su consideración ${ }^{18}$, así como las obras suyas conservadas en las colecciones de Amberes del momento ${ }^{19}$.

La pintura que quisiéramos añadir al catálogo del pintor es obra de gran tamaño conservada en la Colegiata de Santa Gertrudis de la ciudad belga de Nivelles (fig. 1) ${ }^{20}$. Se tiene en la actualidad como anónimo flamenco del siglo XVII, y en antiguos documentos con atribución a Gaspar de Crayer $^{21}$. Los inventarios de la Colegiata de principios del siglo XX informan de que el cuadro procede de la antigua iglesia de Nuestra Señora de Nivelles, principal y primera iglesia parroquial de la ciudad que se hallaba en la Grand Place. El templo fue cerrado hacia 1798 durante la invasión francesa y transformado en vivienda en 1813. Entonces se transfirió parte del mobiliario a la Colegiata, siendo el resto del edificio demolido en $1960^{22}$. Sorprende que las interesantes obras que conserva la Colegiata no hayan llamado la atención de los estudiosos, pues allí reconocimos otras pinturas inéditas y de calidad cuyo estudio preparamos ${ }^{23}$. Es posible que la pintura de Nivelles fuera comanditada por la iglesia parroquial, si bien no hay datos que

16 Viena, Kunsthistorisches Museum, Inv. n. ${ }^{\circ}$ 1679, 1698, 3549, 3551, 1863, 3552 [Véase A. BERGER, "Inventar der Kunstsammlung der Ersherzogs Leoplod Wilhelm von österreicht. Nach der Originalhandschrift im Fürstlich Schwarzenbergschen Centralarchieve herausgegeben", Jahrbuch der kunsthistorischen Sammlungen des Allerhöchsten Kaiserhouses, I, 1883, nos. 786, 787; G. HeINZ, "Studien über Jan van den Hoecke und die Malerei der Niederländen in Wien", Jahrbuch der Kunsthistorischen Sammlungen in Wien, 63, 1967, p. 130; MAUFORT, op. cit., 1986, cat. n. ${ }^{\circ}$ M1, M2 M3, M4, M5, M6, M7; HeInRICH, op. cit., 2003, Cat. nos. A63, A64]. El inventario de la colección del archiduque registra otras dos pinturas suyas, hoy perdidas, con Siete angelitos en un paisaje y Píramo y Tisbe [BERGER, op. cit., 1883, p. 116, n. ${ }^{\circ} 21$; p. 136, n. ${ }^{\circ}$ 397; cit. H. Vlieghe, "Thoughts on Van Dyck's Early Fame and Influence in Flanders", in Van Dyck 350, op. cit., 1994, p. 220, n. 57].

17 Hoy conservado en la Oranjezaal de la Huis Ten Bosch cerca de La Haya [véase E. Duverger, "Abraham van Diepenbeeck en Gonsales Cocques aan het werk vook de stadhouder Frederik Hendrik, prins van Oranje", Jaarboek van het Koninklijk Museum loor Schone Kunsten Antwerpen, 1972, p. 183; Maufort, op. cit., 1986, cat. n. ${ }^{\circ}$ M10].

18 P. F. Rombouts \& Th. VAN LeRIus, De Liggeren en andere historische archieven der Antwerpsche Sint Lucasgilde, Amberes-La Haya, 1864-1876, II, pp. 323, 328, 330; E. Duverger, Antwerpse kunstinventarissen uit de zeventiende eeuw, Bronnen voor de Kunstgeschiedenis van de Nederlanden, IX, p. 502.

19 Duverger, Antwerpsche Kunstinventarissen..., op. cit., vol. VII, pp. 197, 198; vol. VIII, pp. 470, 471; vol. IX, pp. 93, 150, 237, 393, 502; vol. X, pp. 175, 212, 219, 232, 233, 311; vol. XI, pp. 309, 326, 352, 571, 572, 573; vol. XII, pp. 15, 29, 364. A propósito del encargo para la Kolveniersgilde véase N. DE POORTER, "Rubens onder wapenen. De Antwerpsche schilders als gilderbroeders van de kolveniers in de eerste helft van de 17de eeuw", Jaarboeck van het Koninklijk Museum voor Schone Kunsten Antwerpen, 1988, pp. 237-239.

${ }^{20}$ Lienzo, $260 \times 190 \mathrm{~cm}$. Nivelles, Collégiale Sainte-Gertrude.

21 "Saint Dominique recevant le rosaire, tableau de l'école flamande, atribué à Crayer" [cit. Inventaire des objets d'art existant dans les édifices publics des communes de l'arrondissement de Nivelles, Comité des correspondants de la Commission Royale des Monuments et Sites de la Province du Brabant, Bruselas, 1912, p. 8]. Posteriormente lo recoge el inventario del mobiliario de la Colegiata de Santa Gertrudis del canónigo René Mary, decano de Nivelles:

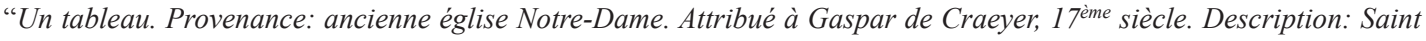
Dominique recevant le Rosaire. Emplacement: Fonds Baptismaux" [René MARY, Inventaire du mobilier de la Collégiale Ste Gertrude, Nivelles, 1938, p. 11]. Información facilitada por M. OsterRIETH, Conservadora del Museo Comunal de Nivelles, a quien expreso mi agradecimiento.

22 Cit. M. Osterrieth, en comunicación escrita.

${ }^{23}$ Una Cena de los Apóstoles y un San Francisco en éxtasis de Gaspar de Crayer, y un Martirio de San Adrián y un Santo Domingo dando la comunión en Oriente de Cornelius Schut; así como una interesante pintura con San Carlos Borromeo y los apestados que recuerda el estilo de Gaspar de Crayer en su primera juventud. 


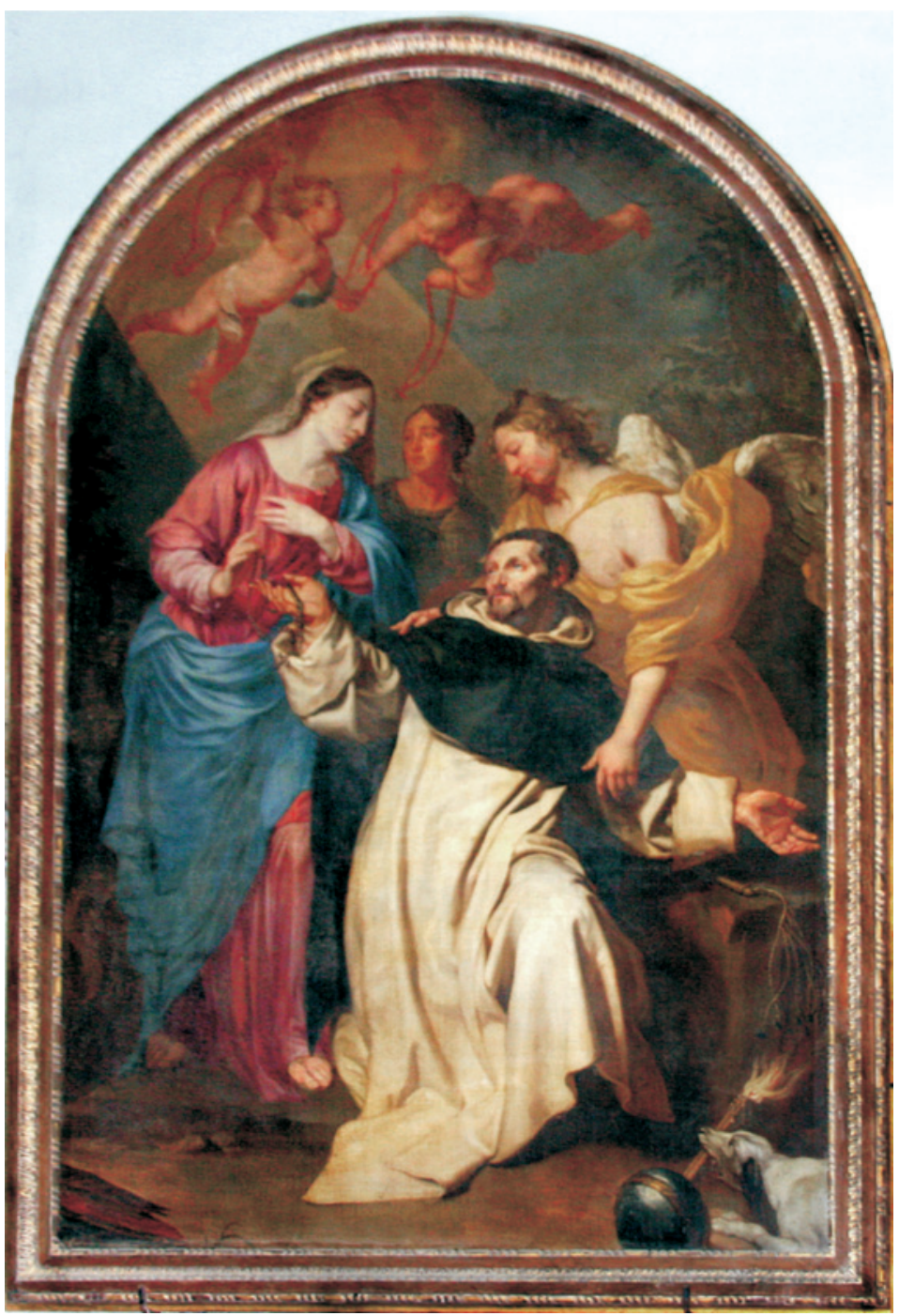

Fig. 1. Peter Thijs. Santo Domingo de Guzmán recibiendo el rosario de la Virgen. Nivelles, Colegiata de Santa Gertrudis.

corroboren esta procedencia en el siglo XVII. Pero una copia antigua que conserva la misma Colegiata (fig. 3$)^{24}$ invita a pensar en este supuesto, y habla a la vez de la categoría que se vio en el original, merecedor de un duplicado que registrara su preexistencia. No obstante la calidad de la copia es visiblemente muy inferior.

Antes de exponer las razones que justifican la atribución a Peter Thijs es necesario detenernos en el asunto representado: Santo Domingo de Guzmán recibiendo el rosario de la Virgen,

${ }^{24}$ Tabla, $69 \times 51 \mathrm{~cm}$. Nivelles, Colegiata de Santa Gertrudis. El soporte es sobre tabla y no sobre lienzo como indica el IRPA. Fotografía de P. Godart. 


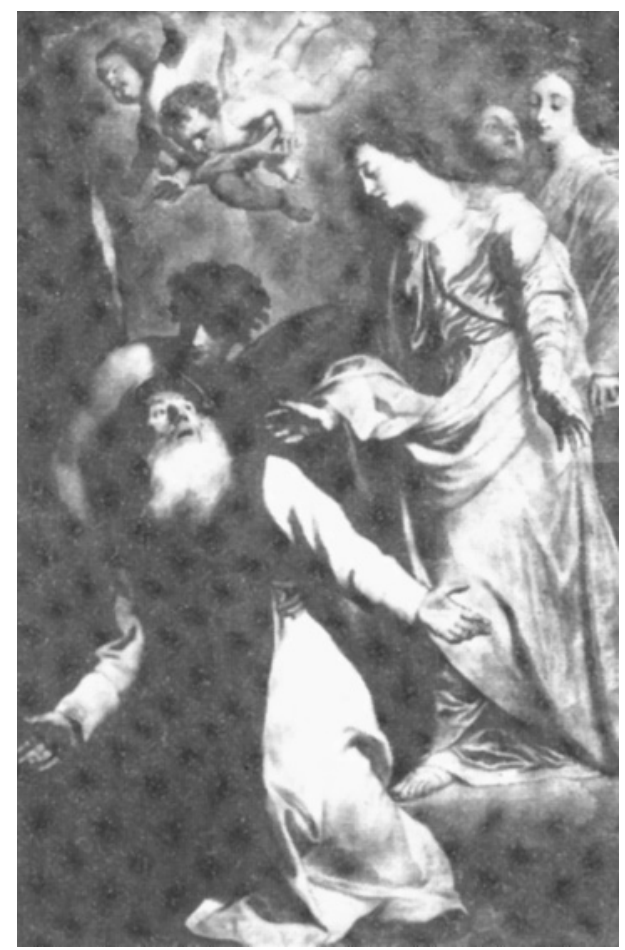

Fig. 2. Peter Thijs. Aparición de la Virgen a San Guillermo de Aquitania. Amberes, Museo Real de Bellas Artes.

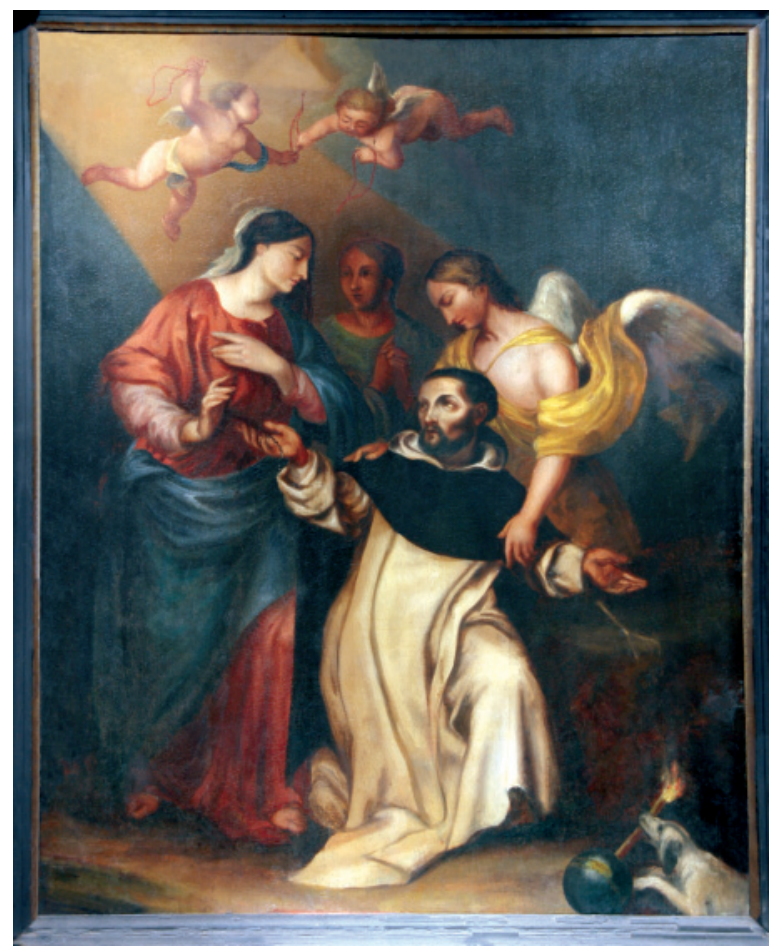

Fig. 3. Copia según Peter Thijs. Santo Domingo de Guzmán recibiendo el rosario de la Virgen. Nivelles, Colegiata de Santa Gertrudis.

uno de los episodios más conocidos de la vida del santo burgalés que fue canonizado en 1234 , tan sólo una década después de su muerte. Es fácil reconocerlo por la sotana blanca y el manto negro que componen el hábito de su orden, cuyos colores simbolizan la pureza y la austeridad; pero también por el rosario, uno de sus más frecuentes atributos, así como por el perro con una antorcha en llamas en la boca, motivo que hace referencia a la leyenda de la visión que habría tenido su madre antes de su alumbramiento, y cuyo origen está en los hechos milagrosos relatados por sus dos principales biógrafos, Jordán de Sajonia, el primer sucesor de Santo Domingo en la orden, y el beato Pedro Ferrando ${ }^{25}$. El perro se interpreta como símbolo del predicador, y su ladrido como voz de la doctrina. Para Pedro Ferrando el ladrido es símbolo de los heréticos, que veía como lobos disfrazados ${ }^{26}$. La escena aquí representada se refiere al episodio en que la Virgen se le apareció en Albi, haciéndole entrega del rosario, arma poderosa para salvar almas en sus predicaciones. No hay mención de éste como atributo suyo en las fuentes literarias; se trata de una leyenda popular sin fundamento histórico de finales de la Edad Media, que fue favorita de los artistas del barroco.

25 Véanse las biografías de Jordán de Sajonia y Pedro Ferrando en M. Gelabert \& J. M. Milagro, Santo Domingo de Guzmán visto por sus contemporáneos, 2. ${ }^{a}$ ed., Madrid, 1966. Véanse también M. H. Vicaire, Histore de Saint Dominique, París, 1957; L. Galmes \& T. Gómez Vito, Santo Domingo de Guzmán: Fuentes para su conocimiento, Madrid, 1987; D. Iturgaiz, Iconografía de Santo Domingo de Guzmán: La fuerza de la imagen, Burgos, 1992.

${ }^{26}$ L. RÉAU, Iconographie de l'Art Chrétien, París, 1958, tome III, Saints, I, p. 391. 
Había en Amberes una larga tradición de fervor al rosario, especialmente impulsada en el primer tercio del siglo XVII por los dominicos y los jesuitas. Las obras de Vincent Herinsbergen y Petrus Vloers contribuyeron a la propagación de esta devoción ${ }^{27}$. El interior de la Iglesia de San Pablo, por entonces templo de los dominicos, desplegaba toda una iconografía en relación con el culto al rosario, práctica usual para combatir las epidemias ${ }^{28}$. Un retablo de Caravaggio con la Madonna del Rosario, hoy en el Kunsthistorisches de Viena, decoraba la iglesia, así como un ciclo dedicado a sus Misterios (aún in situ) en el que colaboraron varios pintores bajo la dirección de Rubens hacia 1617; y escenas de Bonaventura Peeters conmemorando las victorias contra los turcos, obtenidas por los cristianos gracias al poder del rosario ${ }^{29}$.

Desde el punto de vista de la composición la pintura que estudiamos puede aproximarse a algunos precedentes. Eminentemente vandyckiano es el gesto del Santo Domingo, con los brazos extendidos y las palmas hacia arriba, igual que en el lienzo de Van Dyck con Santo Domingo y Santa Catalina ante Cristo en la Cruz que estaba en el Convento de Santo Domingo y hoy conserva el Museo de Amberes $^{30}$. Pero más próximas son las sugestiones del Éxtasis de San Agustín de Van Dyck del Museo de Amberes, pintura que se colocó hacia 1628 en el altar izquierdo de la Iglesia de San Agustín ${ }^{31}$. La composición general guarda similitudes con Los desposorios místicos del beato Hermann Joseph de Van Dyck en el Kunsthistorisches de Viena ${ }^{32}$. Recuerda el modelo de perfil y disposición del cuerpo de la Virgen, así como el ángel que asiste al santo, con las alas desplegadas y el atuendo de vaporosos mantos. Esta pintura decoraba hasta $1773 \mathrm{el}$ Oratorio de la Cofradía de Solteros de los jesuitas de Amberes ${ }^{33}$, una casa que sin duda conocería el pintor. De Van Dyck toma la elegancia y la distinción aristocrática en los gestos de los personajes, eliminando cualquier signo de patetismo.

Al margen de estas sugestiones, Peter Thijs se distancia aquí de las representaciones más frecuentes del tema, en las que la Virgen figura sobre un trono, depositando el rosario en manos de Santo Domingo, en compañía de otros santos en actitudes suplicantes. Reduce los acompañantes al ángel que sostiene al santo en éxtasis y a una figura femenina detrás. La introducción de estos perfiles diluidos en los segundos planos y con actitud desviada de lo que acontece en la escena, es recurso que utiliza con frecuencia el pintor. Las mismas expresiones congeladas pueden verse en numerosas pinturas de su mano, como Mercurio y Herse del Palacio de Wörtlitz ${ }^{34}$ y su versión ampliada del

27 Vincent Heinsbergen, Viridarium Marianum, Amberes, 1626 (con grabados de Boece à Bolswert); Petrus Vloers, véase J. PaQuot, Mémoires pour servir à l'histoire des dix-sept Provinces des Pays-Bas, de la Principauté de Liège et de quelques contrées voisines, Lovaina, 1765-1770, Historiae Litt, XVIII, 307, Diercxsens, Antwerpiae VII, 370, Steph Axters O.P. in Ons Geestelijk Erf, 7, 1933, 282 f. (n. 615-619); Cit. J. B. KNIPPING, Iconography of the Counter Reformation in the Netherlands, Leiden, 1974, p. 278.

${ }^{28}$ KNIPPING, op. cit., 1974, p. 138.

${ }^{29}$ En tiempos de José II el lienzo de Caravaggio fue remplazado por una copia (J. DE WITT, De kerken van Antwerpen, II, fol. 5r, fol. 29r; cit. KNIPPING, op. cit. 1974, p. 278, 159. A propósito del ciclo de los Misterios del Rosario, véase R. SiRJACoBS, Antwerpen Sint Pauluskerk: Rubens en de mysteries van de rozenkrans, Amberes, 2004.

30 Véase S. Barnes, N. De Poorter, O. Millar \& H. Vey, Van Dyck. A complete catalogue of the paintings, Londres-New Heaven, 2004, p. 267, n. ${ }^{\circ}$ III.28.

${ }^{31}$ Lienzo, $398 \times 227 \mathrm{~cm}$. Amberes, Koninklijk Museum Voor Schone Kunsten [véase S. BARNES et al., op. cit. 2004, p. 277, n. ${ }^{\circ}$ III.47].

${ }^{32}$ Lienzo, $275 \times 210 \mathrm{~cm}$. Viena Kunsthistorisches Museum, Inv. n. ${ }^{\circ} 482$ [S. BARNES et al., op. cit. 2004, p. 283, n. ${ }^{\circ}$ III.49].

${ }^{33}$ Véase W. SCHEELEN, "Het lot van de schilderijencollecties van de Zuidnederlandse Jezuïtencolleges na de opheffing van de Orde in 1773", Jaarboeck van het Koninklijk Museum voor Schone Kunsten Antwerpen, 1988, p. 295.

${ }^{34}$ Lienzo, $183 \times 151 \mathrm{~cm}$. Firmado y fechado: Peter Thijs Fecit 1664. Wörlitz, Staatliche Schlösser und Gärten [véase HAIRS, op. cit. 1977, p. 268; MAUFORT, op. cit. 1986, cat. n. ${ }^{\circ}$ M17]. 
Herzog Anton Ulrich Museum de Brunswick ${ }^{35}$, el Emperador Heraclio llevando la Santa Cruz de la Iglesia de Zwijndrecht ${ }^{36}$, Aquiles descubierto por Ulises en casa de Lycomedes del Museo de Estocolmo ${ }^{37}$, y versión que tuvo A. Rosenthal en Londres ${ }^{38}$, así como la Aparición de la Virgen a San Guillermo de Aquitania del Museo de Amberes, pintura que lleva la firma Peeter Thijs Fecit (fig. 2) ${ }^{39}$. El cotejo con esta última es fundamental para avalar nuestra hipótesis de autoría, pues comparte con el lienzo que nos ocupa espíritu y composición, concepción del espacio, modelos y colorido. Similar es la actitud del santo con los brazos extendidos, los rostros ovalados, el perfil de ojos bajos de la Virgen, su silueta elegante con flemática actitud y delicados gestos, la concepción de los pliegues de los mantos, así como los ángeles que revolotean en la parte superior. El ángel mancebo con las alas desplegadas e inspirado en la pintura de Van Dyck más arriba mencionada, se asemeja al que acompaña a la Virgen de la Porciúncula del Museo de Amberes (fig. 4) ${ }^{40}$. Obsérvese en ésta la mano en el pecho de la Virgen y el angelito en la parte superior, repetido en la pintura de Nivelles que nos ocupa. El rosado intenso de la túnica de la Virgen, el azul del manto y el ocre amarillo del ángel son los colores que constituyen su paleta habitual.

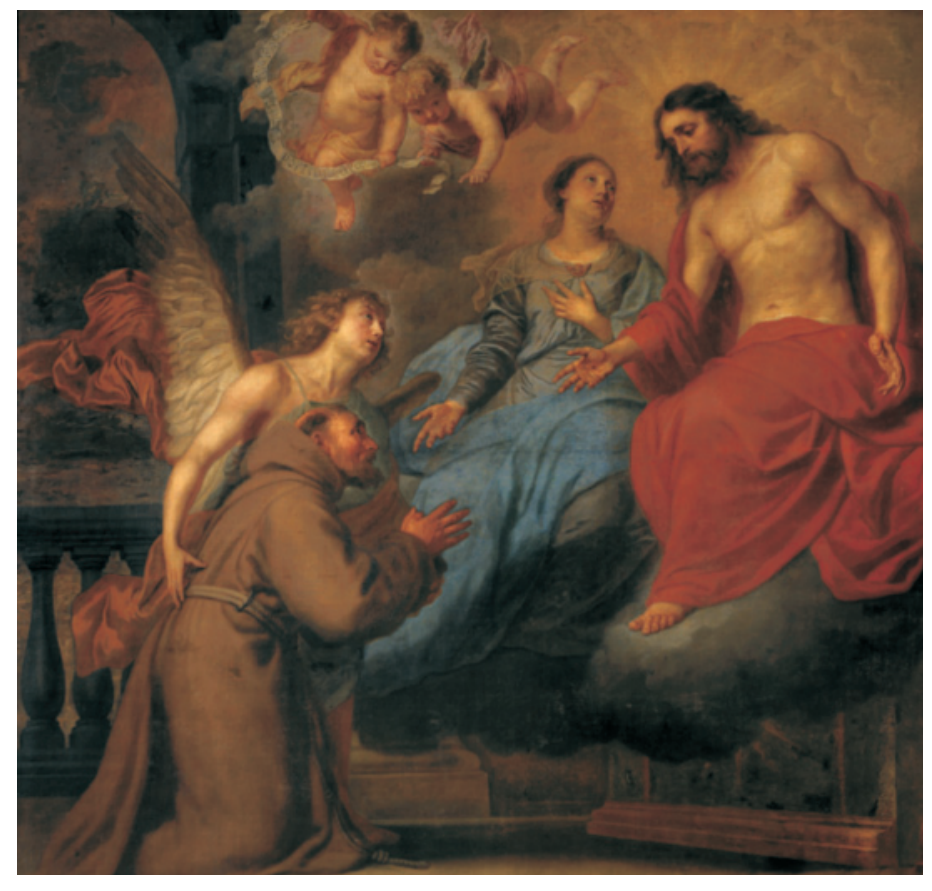

Fig. 4. Peter Thijs.

Virgen de la Porciúncula. Amberes, Museo Real de Bellas Artes.

35 Lienzo, $160 \times 244 \mathrm{~cm}$. Brunswick, Herzog Anton Ulrich Museum, Inv. n. ${ }^{\circ} 1063$. Atribuido en los catálogos del Museo a Théodoor van Thulden hasta 1977. [Véase HAIRs, op. cit., 1977, p. 274; Maufort, op. cit., 1986, cat. n. $\left.{ }^{\circ} \mathrm{M} 23\right]$.

${ }^{36}$ Lienzo, $210 \mathrm{~cm}$ de ancho. Zwijndrecht, Iglesia de la Crucifixión. [Véase HaIRS, op. cit., 1977, p. 269, n. 29; MAUFORT, op. cit., 1986, cat. n. ${ }^{\circ}$ R24].

${ }^{37}$ Lienzo, $199 \times 234 \mathrm{~cm}$. Estocolmo, Nationalmuseum, Inv. n. ${ }^{\circ} 662$ [véase G. GöTHE, Notice descriptive des tableaux du Musée National de Stockolm, I, Maîtres étrangers, Estocolmo, 1893, p. 318, n. ${ }^{\circ} 622$; MAUfORT, op. cit., 1986, cat. n. $\left.{ }^{\circ} \mathrm{M} 18\right]$.

38 Lienzo, 152,2 $\times 203 \mathrm{~cm}$. Paradero actual desconocido [vid. MAUfORT, op. cit., 1986, cat. n. ${ }^{\circ}$ M22].

39 Lienzo, $249 \times 167 \mathrm{~cm}$. Firmado: Peter Thijs Fecit. Amberes, Koninklijk Museum loor Schone Kunsten, Inv. n. ${ }^{\circ} 354$ [HAIRS, op. cit., 1977, p. 268; MAUFORT, op. cit., 1986, cat. n. ${ }^{\circ}$ R22].

${ }^{40}$ Lienzo, $248 \times 263 \mathrm{~cm}$. Amberes, Koninklijk Museum voor Schone Kunsten, inv. n. ${ }^{\circ}$ 352. [Véase HaIrs, op. cit., 1977, p. 271; Douglas Stewart, op. cit., 2001, p. 277, fig. 8; Maufort, op. cit. 1986, cat. n. ${ }^{\circ}$ r16]. 
Estos caracteres estilísticos corresponden a la época de madurez de Peter Thijs, en la segunda mitad de la década de $1660^{41}$, donde cabe situar esta pintura. El pintor está en el apogeo de su carrera. Su nombramiento como Decano de la guilda de pintores en 1661 habla de la fama que había adquirido por entonces. Su éxito le proporcionó en esta década y hasta su muerte numerosos encargos oficiales del clero para iglesias y conventos. En esta época madura de su evolución Peter Thijs suele servirse, como aquí, de composiciones piramidales y simétricas, y vestir a sus figuras femeninas con túnicas atadas a la cintura y mantos formando pliegues en V. La tela se adhiere al muslo y a la rodilla, dando lugar a un elaborado juego de finos pliegues. En la indumentaria masculina, en cambio, los pliegues suelen ser más amplios, plasmando con exactitud las diferentes texturas de las telas, el satén y el algodón ${ }^{42}$.

La mano de Thijs se reconoce bien en el lienzo de Santo Domingo recibiendo el rosario de la Virgen en los rostros de corte ovalado, en los ojos de forma almendrada, en el corte del arco de las cejas, en las arrugas a ambos lados de las aletas de la nariz, y en el cuello robusto, rasgos recurrentes en sus modelos ${ }^{43}$.

La tipología de los modelos de Thijs es tan afín a la de Willeboirts Bosschaert que la atribución de algunas obras a uno y otro pintor es cuestión que sigue abierta ${ }^{44}$. De hecho, se ha sugerido que Thijs fuera discípulo de Willeboirts, unos diez años mayor que él, al ver la inspiración que toma de sus obras ${ }^{45}$. Ambos deben a Van Dyck la elegancia y las actitudes de los personajes, tornándose a precedentes italianos de Tiziano, Veronés y Reni ${ }^{46}$. Sus modelos son de inspiración clásica, sus siluetas robustas y los rostros, con carnaciones de porcelana, tienen una expresión extática. No obstante, como ya señaló D. Maufort, el rasgo diferenciador fundamental para deslindar sus estilos reside en la pincelada más suelta de Willeboirts y en la más acusada humanidad y sensualidad que desprenden sus modelos, en especial las figuras femeninas. La materia en Thijs es en cambio más apretada y espesa, y sus modelos expresan la emoción con mayor decoro y contenida teatralidad ${ }^{47}$.

JAHEL SANZSALAZAR*

41 Véase el estilo de Peter Thijs en la década de 1660 a 1670 que analizó D. MAUfort [op. cit., 1986, sp, Hoofstuk 3, De stijlevolutie van Peeter Thijs, (3: Van ca. 1669 tot 1669)].

42 Véase Maufort, op. cit., 1986, p. 32.

43 Véase MAufort, op. cit., 1986, p. 27.

${ }^{44}$ Véase lo dicho en notas 12 y 13.

45 Vlieghe, op. cit., 1994, p. 214.

${ }^{46}$ MAUfORT, op. cit., 1986, p. 25.

47 Cit. MAuforT, op. cit., 1986, pp. 26, 31.

* Quisiera expresar mi sincero agradecimiento a la Sra. Martine Osterrieth, del Musée Communal de Nivelles, por su extrema amabilidad y mejor disposión para facilitarme todo lo necesario al estudio de la pintura, al Sr. Philippe Dardenne, responsable del Patrimonio de la Fábrica de la Iglesia de la Colegiata, y al Sr. Philippe Godart, del Royal photo club "Entre nous", a quien se deben las fotografías. 